\title{
Alterações Cardiovasculares na Síndrome da Apnéia Obstrutiva do Sono
}

\author{
Cardiovascular Comorbidities and Obstructive Sleep Apnea
}

Fátima Dumas Cintra, Dalva Poyares, Christian Guilleminault, Antonio Carlos Carvalho,
Sergio Tufik, Angelo A. V. de Paola
Universidade Federal de São Paulo e Stanford University - São Paulo, SP - Stanford, CA, USA

A síndrome da apnéia obstrutiva do sono (SAHOS) é uma condição prevalente na população, associada a maior risco cardiovascular, freqüentemente não-diagnosticada. 0 reconhecimento da síndrome requer alto grau de suspeita clínica, especialmente por cardiologistas, e pode ser confirmada por meio da polissonografia. O tratamento da síndrome com o uso de CPAP (pressão positiva na via aérea superior) é altamente eficaz, melhorando o padrão respiratório durante o sono, instituindo o sono reparador e, dessa forma, otimizando a qualidade de vida desses pacientes, além de atenuar ou reverter muitas das complicações cardiovasculares relacionadas a SAHOS. Este artigo aborda a fisiopatologia e os aspectos clínicos das comorbidades cardiovasculares associadas à síndrome.

\section{INTRODUÇÃo}

A doença cardiovascular é uma das principais causas de mortalidade no mundo ${ }^{1}$. Na cidade de São Paulo, as doenças cardiovasculares associadas à aterosclerose representam a principal causa de óbito semelhante aos países desenvolvidos ${ }^{2,3}$. Vários estudos ${ }^{4-6}$ confirmam a importância do tabagismo, níveis elevados de LDLcolesterol, baixos níveis de HDL-colesterol, diabete melito, hipertensão arterial sistêmica, história familiar, obesidade, sedentarismo, obesidade central, síndrome plurimetabólica e ingesta de álcool na gênese da aterosclerose e suas complicações clínicas.

Além desses fatores, têm sido observado recentemente evidências de aumento na taxa de mortalidade cardiovascular em pacientes portadores da síndrome da apnéia/hipopnéia obstrutiva do sono (SAHOS) 7 . 0 papel da síndrome como fator de risco cardiovascular merece muita atenção do cardiologista, uma vez que se trata de uma condição freqüentemente não-diagnosticadå .

A SAHOS é caracterizada pela obstrução completa ou parcial recorrente das vias aérea superiores durante o sono, resultando em períodos de apnéia, dessaturação de oxiemoglobina e despertares noturnos freqüentes (figs. 1 e 2) com conseqüente sonolência diurna. Apresenta predileção pelo sexo masculino, e a incidência nos homens e mulheres de meia-idade varia na literatura entre $1 \%$ e $5 \%$ e $1,2 \%$ e $2,5 \%$, respectivamente ${ }^{9-13}$, podendo aumentar com a idade ${ }^{14}$. Associa-se freqüentemente a outras doenças cardiovasculares, estimando-se que $40 \%$ dos pacientes portadores de hipertensão arterial sistêmica apresentam SAHOS associada, não-diagnosticada e não-tratada ${ }^{15,16}$.

O quadro 1 mostra algumas definições importantes para interpretação e diagnóstico dessa entidade.

\section{Fisiopatologia}

Maior colapso das vias aéreas superiores durante o sono está associado a maior esforço respiratório e alteração no fluxo nasal e oral, que podem desencadear hipoxemia e hipercapnia ${ }^{20}$. Esse processo leva a um despertar com restabelecimento da ventilação. A alternância entre os eventos respiratórios anormais e os despertares pode ocorrer várias vezes durante a noite ${ }^{21}$.

Durante cada episódio de apnéia/hipopnéia obstrutiva a inspiração forçada contra a via aérea ocluída é acompanhada de pressão negativa no espaço pleural. À medida que a apnéia se prolonga, acentuam-se a hipoxemia e a hipercapnia, levando a vasoconstrição pulmonar, com o desenvolvimento de hipertensão pulmonar transitória. Ocorre, por sua vez, estimulação do sistema nervoso simpático, com vasoconstrição sistêmica e hipertensão arterial, e em alguns casos a pressão arterial sistólica pode alcançar níveis noturnos significativamente elevados, mesmo em indivíduos com pressão arterial normal durante a vigília ${ }^{22}$.

Além disso, o fenômeno de hipoxemia e subseqüente reoxigenação, repetido por inúmeras vezes durante a noite, ocasiona alterações de reperfusão com formação de radicais livres ${ }^{23}$, e o estresse oxidativo é atualmente considerado um contribuinte importante para as conseqüências cardiovasculares observadas nesse grupo de pacientes ${ }^{24}$. A associação da SAHOS à obesidade ${ }^{25}$, o predomínio pelo sexo masculino, e feminino após a menopausa, bem como os efeitos sistêmicos desencadeados com o seu aparecimento sugerem fortemente que a SAHOS é uma doença sistêmica e 


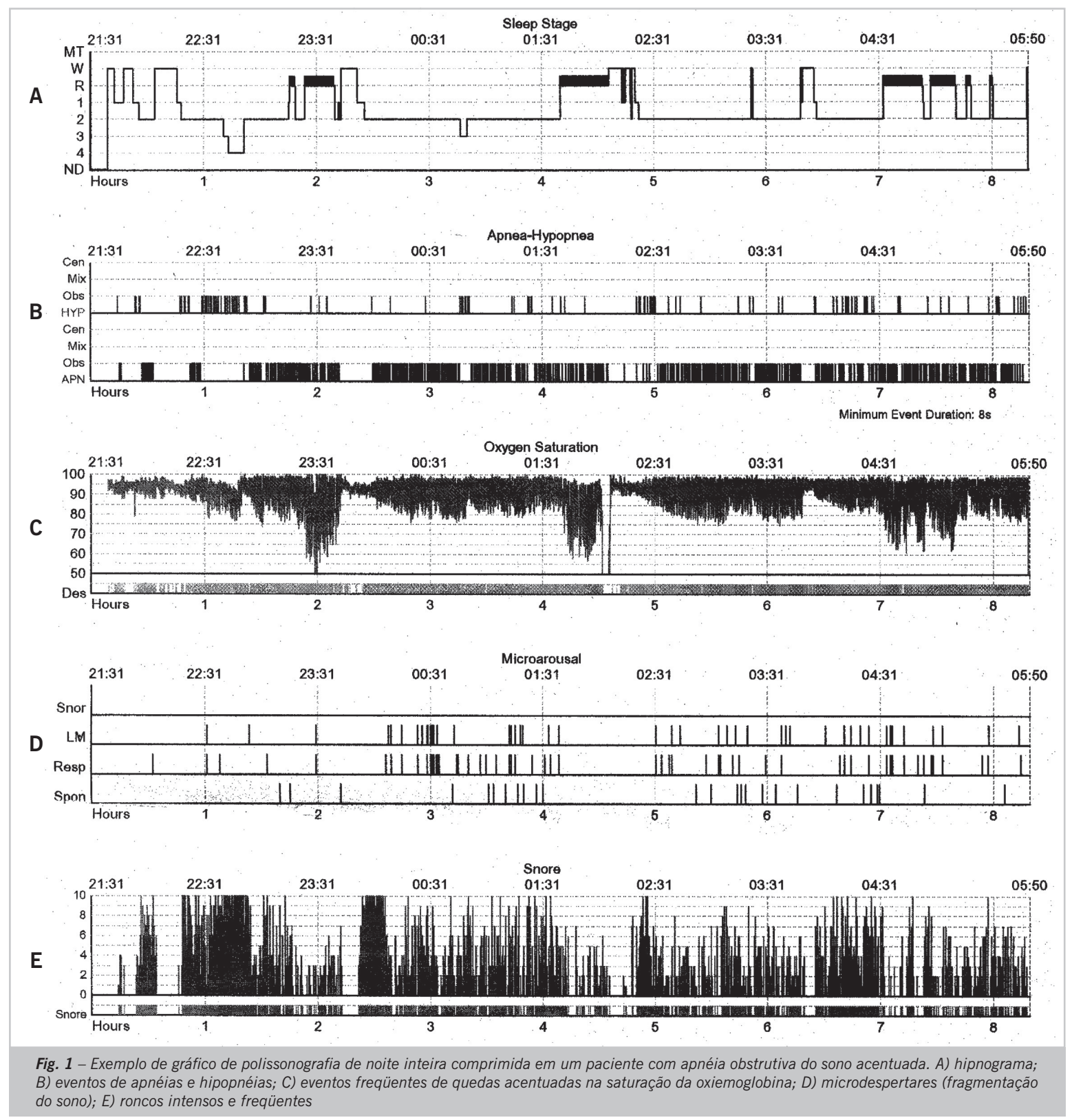

não uma anormalidade local ${ }^{26,27}$. Outras evidências importantes que favorecem a interpretação da SAHOS como uma doença sistêmica são as suas correlações com o aumento das citocinas inflamatórias e com a resistência a insulina. As citocinas inflamatórias, como o fator- $\alpha$ de necrose tumoral (TNF- $\alpha$ ) e a interleucina 6 (IL-6), estão envolvidas na regulação fisiológica do sono ${ }^{28}$ e encontram-se anormalmente elevadas em pacientes apnéicos quando comparados com indivíduos normais e com pacientes portadores de obesidade ${ }^{29,30}$. Entretanto, a correlação entre a SAHOS e a resistência à insulina parece ser independente da obesidade ${ }^{31}$.

\section{CONSEQÜÊNCIAS CARDIOVASCULARES}

As principais conseqüências cardiovasculares são: alterações no sistema nervoso autonômico, hipertensão arterial, arritmias cardíacas, doença arterial coronária, acidente vascular cerebral e insuficiência cardíaca congestiva.

\section{Alterações no sistema nervoso autônomo}

As alterações na freqüência cardíaca e pressão arterial, observadas na apnéia obstrutiva durante o sono, são provavelmente secundárias a alterações no sistema nervoso autônomo durante cada evento apneico ${ }^{32}$, o que foi demonstrado mesmo em pacientes com SAHOS de grau leve ${ }^{33,34}$. Durante esses eventos observa-se um progressivo aumento na atividade simpática, atingindo o seu pico máximo no término da apnéia, seguido por uma 


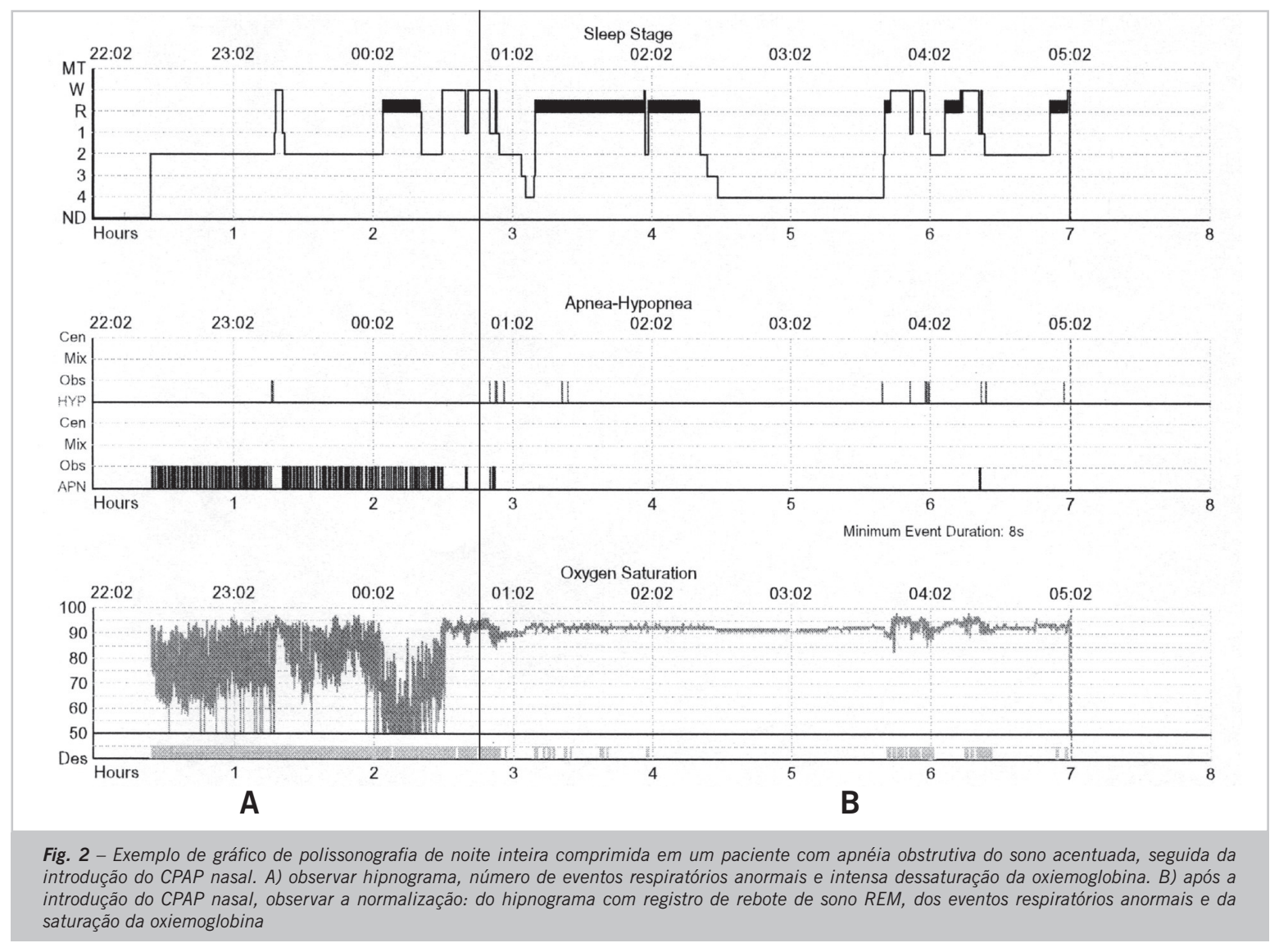

\begin{tabular}{|c|c|}
\hline \multicolumn{2}{|r|}{ Quadro 1 - Definição de termos ${ }^{17-19}$} \\
\hline Termo & Definição \\
\hline Polissonografia & $\begin{array}{l}\text { Registro poligráfico do sono incluindo: registro do eletroencefalograma, eletrooculograma, eletromiograma, } \\
\text { eletrocardiograma e parâmetros respiratórios. }\end{array}$ \\
\hline Sono NREM & Caracterizado por ondas eletroncefalográficas lentas associado a grafoelementos específicos. \\
\hline Sono REM & $\begin{array}{l}\text { Caracterizado por dessincronização eletroencefalográficas, movimentos rápidos dos olhos, atonia muscular } \\
\text { e sonhos. }\end{array}$ \\
\hline Despertares & Despertar transitório durante o sono que duram mais de três segundos. \\
\hline Apnéia & Redução $>80 \%$ do fluxo aéreo oronasal por mais de dez segundos. \\
\hline Hipopnéia & $\begin{array}{l}\text { Redução }>20 \% \text { do fluxo aéreo oronasal por mais de dez segundos, geralmente associada com uma } \\
\text { redução na saturação da oxiemoglobina ou a um despertar. }\end{array}$ \\
\hline Índice apnéia-hipopnéia (IAH) & Freqüência de apnéia e hipopnéia por hora de sono; uma medida da graduação da apnéia do sono., \\
\hline Dessaturação do oxigênio & Quedas $>3 \%$ na saturação da oxiemoglobina, freqüentemente como resultado da apnéia ou hipopnéia. \\
\hline $\begin{array}{l}\text { Síndrome da apnéia e hipopnéia } \\
\text { obstrutiva do sono }\end{array}$ & $\begin{array}{l}\text { IAH > cinco por hora de sono associado a sintomas como roncos, sono agitado, dispnéia noturna, } \\
\text { cefaléias pela manhã, sonolência excessiva diurna e hipertensão arterial. }\end{array}$ \\
\hline
\end{tabular}

acentuada diminuição durante a recuperação ${ }^{35,36}$.

Os mecanismos pelos quais a SAHOS determina ativação simpática persistente, ou seja, mesmo durante a vigília, entretanto, ainda não são completamente conhecidos. Existem algumas evidências que enfatizam o papel da hipóxia nesse fenômeno: as concentrações urinárias de norepinefrina são inversamente proporcionais à saturação da oxiemoglobina mínima noturna. Além disso, as mudanças ventilatórias que determinam hipóxia e hipercapnia causam elevação da atividade muscular simpática, que pode persistir por até vinte minutos após a retirada do estímulo ${ }^{37}$. O seio carotídeo parece desempenhar um papel no processo entre a exposição à hipóxia intermitente noturna e o desenvolvimento da elevação sustentada nos níveis de ativação autonômica, uma vez que a regulação e a sensibilidade barorreflexa estão diminuídas em pacientes com SAHOS, comparados com controles ${ }^{38}$. Evidências atuais sugerem que o estímulo hipóxico intermitente noturno dos quimioceptores periféricos aumentam o tônus simpático em tais pacientes mesmo em vigília e em condições ideais de suprimento de oxigênio ${ }^{39,40}$. 
Além disso, estudos do sistema nervoso autônomo por meio da variabilidade da freqüência cardíaca mostram que pacientes portadores de SAHOS apresentam um decréscimo no componente de alta freqüência (tônus parassimpático) da variabilidade da freqüência cardíaca e um aumento no componente de baixa freqüência (tônus simpático). Esse padrão anormal parece refletir uma predominância simpática na modulação autonômica da SAHOS ${ }^{41}$.

\section{Hipertensão}

SAHOS é fator de risco independente para hipertensão arterial sistêmica (HAS) ${ }^{42-47}$. A prevalência da HAS em portadores da SAOS varia de $40 \%$ a $90 \%$, e o inverso, a prevalência da SAOS entre portadores de HAS, é de $22 \%$ a $62 \%{ }^{43}$. Estudos recentes confirmam essa alta prevalência entre ambas as condições ${ }^{48}$ e mostram que, em um período de quatro anos, aqueles indivíduos com um índice de apnéia/hipopnéia (IAH) maior que quinze por hora de sono têm um risco três vezes maior (45\% de probabilidade) de desenvolver $\mathrm{HAS}^{43}$. Um estudo de 44 pacientes portadores de HAS resistente à medicação mostrou que $83 \%$ apresentaram SAHOS ${ }^{49}$.

Pacientes com SAHOS têm grande variabilidade da pressão arterial (PA) durante a noite, podendo não apresentar o descenso noturno observado em indivíduos normais, levando a um valor médio noturno elevado, mesmo nas situações em que se observam níveis pressóricos normais durante o dia. Esse comportamento da PA pode decorrer dos seguintes fatores: pressão intratorácica negativa com redução do débito cardíaco e ativação diferencial de barorreceptores, hipóxia, hipercapnia, despertar do sono relacionado ao evento respiratório e aumento da atividade simpática ${ }^{50}$.

O CPAP (contínuos positive airway pressure) tem se mostrado eficaz no tratamento dos pacientes portadores de SAHOS e HAS ${ }^{51,52}$. Em uma casuística de onze pacientes com HAS refratária, o uso do CPAP reduziu os níveis pressóricos noturnos ${ }^{53}$. Além disso, em doze pacientes com SAHOS grave, o uso do CPAP reduziu a norepinefrina plasmática e os metabólicos urinários das catecolaminas ${ }^{54}$.

Até o momento atual, portanto, a SAHOS deve ser considerada no diagnóstico diferencial de causas de hipertensão arterial sistêmica refratária e o uso do CPAP poderia auxiliar na redução das pressões arteriais noturnas e diurnas ${ }^{55,56}$.

\section{Arritmias cardíacas}

A relação entre a SAHOS e as arritmias cardíacas já foi analisada na literatura médica por alguns autores ${ }^{57,58}$, não havendo consenso nos resultados encontrados e na prevalência das taquiarritmias e das bradiarritmias. Esse fato pode ser em parte explicado pela desconhecida incidência de SAHOS na população geral saudável e pela alta incidência de hipertensão e doenças cardiovasculares em pacientes com SAHOS. Entretanto, sabe-se que o uso do CPAP em pacientes com SAHOS pode diminuir a incidência de arritmias cardíacas, assim como a estimulação cardíaca artificial pode diminuir a gravidade dos distúrbios respiratórios ${ }^{59,60}$. A figura 3 mostra exemplo de pausa sinusal associada a um evento de apnéia em um paciente portador de SAHOS.

A fibrilação atrial (FA) merece destaque por apresentar maior número de estudos clínicos com os distúrbios respiratórios do sono61-66. A prevalência de FA parece aumentar em pacientes com SAHOS e insuficiência cardíaca congestiva ou revascularização miocárdica recente ${ }^{61,62}$. Conforme referido anteriormente, a SAHOS ocasiona hipoxemia intermitente, ativação simpática e alterações abruptas na pressão arterial, razões essas possivelmente relacionadas ao desenvolvimento e recorrência de FA. Em um estudo prospectivo ${ }^{62}$, com pacientes referidos para a cardioversão elétrica de FA/ flutter atrial, observaram-se $82 \%$ de recorrência nos pacientes com SAHOS sem tratamento ou com tratamento inadequado, e $42 \%$ de recorrência nos pacientes tratados. Além disso, no grupo de pacientes não-tratados, a recorrência foi ainda maior entre os que apresentavam maior queda na saturação de oxigênio durante o evento de apnéia. Esses dados sugerem que o tratamento adequado com CPAP pode fazer diminuir a recorrência de FA nos pacientes portadores de SAHOS.

As ectopias ventriculares foram relatadas em até $66 \%$ dos pacientes portadores de SAHOS. Entretanto, em um estudo prospectivo com 147 pacientes submetidos a polissonografia com registro simultâneo de Holter, não foi encontrado aumento de arritmias ventriculares ${ }^{67}$. Os achados discrepantes na literatura médica dificultam o estabelecimento de uma relação direta entre a síndrome e as arritmias ventriculares. Entretanto, quando se analisa a relação entre as ectopias ventriculares e a dessaturação da oxiemoglobina em pacientes com SAHOS ${ }^{68}$, observa-se que ocorre um aumento acentuado na freqüência de ectopias ventriculares quando a saturação de oxigênio cai abaixo de $60 \%$, e a relação entre eventos apnéicos e arritmias ventriculares parece existir apenas nos pacientes que apresentem importante dessaturação durante a noite.

Pacientes portadores da síndrome apresentam arritmias ventriculares predominantemente durante o sono, diferente dos pacientes com sono normal ${ }^{69}$, sendo a taquicardia ventricular mais comum em pacientes com SAHOS (0-15\%) do que na população geral $(0-4 \%)^{69}$. As bradiarritmias são bastante associadas a SAHOS. Guilleminault e cols. ${ }^{70}$ observaram a ocorrência de pausa sinusal $(>2,5$ segundos), bloqueio atrioventricular de segundo grau, e bradicardia sinusal em $11 \%, 8 \%$ e $7 \%$, respectivamente. Koehler e cols. ${ }^{71}$ analisaram os fatores envolvidos nos bloqueios cardíacos em pacientes com SAHOS, concluindo que na maioria dos casos o bloqueio ocorre durante 0 estado de sono REM e durante os períodos com queda na saturação de oxigênio em pelo menos $4 \%$. 


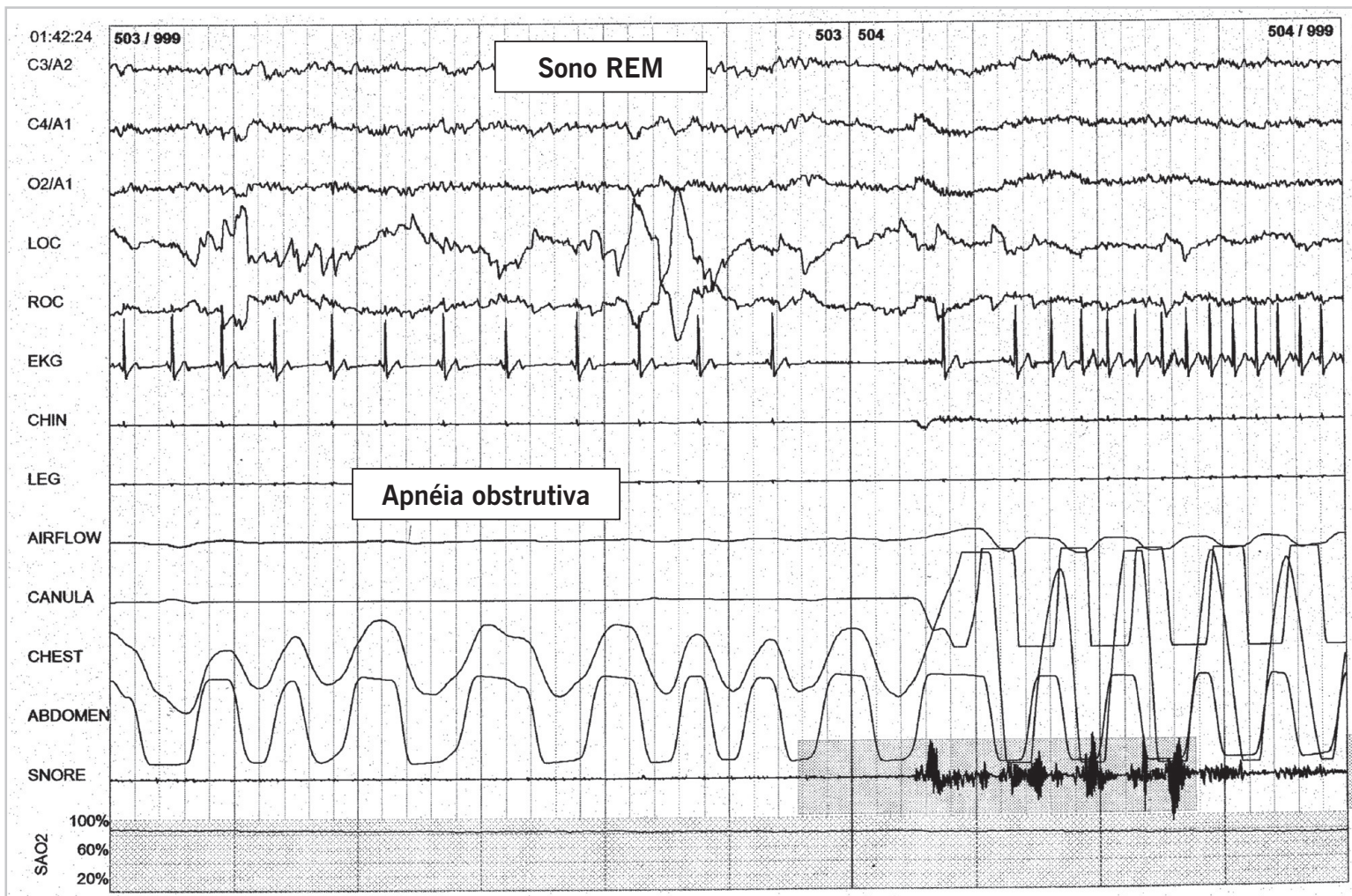

Fig. 3 - Exemplo gráfico de uma época de trinta segundos contendo um evento de apnéia obstrutiva do sono, com pausa sinusal seguida de incremento na freqüência cardíaca

Apesar dos resultados discordantes na literatura, parece que a queda na saturação de oxigênio durante os episódios de apnéia é um importante fator desencadeador de arritmias cardíacas nos pacientes portadores da síndrome e a sua freqüência associa-se à intensidade da hipóxia.

\section{Doença arterial coronariana}

Mesmo com a observação crescente da ocorrência de SAHOS e do concomitante aumento da mortalidade cardiovascular $^{9}$, a maioria dos estudos apresenta limitações importantes, pelo fato de muitos fatores de risco para SAHOS, como obesidade, sexo masculino, idade, dentre outros, serem os mesmos para hipertensão e doença arterial coronária (DAC). Portanto, definir o risco preciso de DAC atribuído a SAHOS parece ser difícil, mas observações convergentes apontam SAHOS como um importante fator associado a DAC.

Na SAHOS o aumento da atividade nervosa simpática periférica durante o sono persiste, durante a vigília, com o dobro dos valores normais ${ }^{72}$, podendo contribuir dessa forma para os eventos coronários agudos nas primeiras horas da manhã. Além do envolvimento do sistema nervoso autônomo, a inflamação e a lesão endotelial, observada na SAHOS, poderiam também participar dos mecanismos envolvidos na $\mathrm{DAC}^{73}$.

Em um estudo bem conduzido avaliando o impacto do tratamento da síndrome na evolução cardiovascular em longo prazo (86,5 \pm 39 meses) de pacientes com DAC, observou-se que os pacientes adequadamente tratados apresentaram redução significativa no risco de ocorrência de eventos cardiovasculares definidos como morte cardiovascular, síndrome coronária aguda, hospitalização por insuficiência cardíaca, ou necessidade de revascularização miocárdica ${ }^{74}$.

Alterações noturnas de segmento ST consistentes com isquemia miocárdica são comuns em pacientes com SAHOS e DAC. Mooe e cols. ${ }^{75}$ avaliaram a ocorrência de isquemia miocárdica noturna e suas relações com as desordens respiratórias do sono. A depressão do segmento ST noturna ocorreu em 31\% dos pacientes analisados, e a associação temporal entre os achados eletrocardiográficos e os episódios de apnéia ocorreram em $19 \%$ dos casos, sendo mais freqüentes em homens $(p<0,01)$ e nas desordens respiratórias mais graves.

\section{Acidente vascular cerebral}

Entre os indivíduos com acidente vascular cerebral (AVC), a incidência de distúrbio respiratório do sono pode ser maior do que $50 \%{ }^{76}$, com predomínio da forma obstrutiva. Ainda permanece pouco claro, entretanto, se esses eventos detectados após o AVC são conseqüência ou uma condição preexistente ao evento cerebrovascular. Da mesma forma que a doença arterial coronária, a SAHOS e o AVC também apresentam muitos fatores de risco em comum, sendo igualmente difícil provar relação causa-efeito. 
Dziewas e cols. ${ }^{77}$ analisaram a freqüência dos distúrbios respiratórios do sono em grupos de pacientes com primeiro episódio e recorrência de AVC isquêmico. Os pacientes com recorrência apresentaram índice de apnéia/hipopnéia médio maior quando comparados com indivíduos com apenas um episódio (26,6/h versus $15,1 / \mathrm{h}, \mathrm{p}<0,05)$ e mais freqüentemente apresentavam SAHOS. Na análise multivariada com ajuste para as variáveis clínicas e fatores de risco, a apnéia do sono foi considerada um fator de risco independente para recorrência de AVC, e os autores advogam o uso da polissonografia nesse grupo de pacientes como forma de estratificação de risco.

Provavelmente a SAHOS contribui para a ocorrência de AVC por vários mecanismos: hipertensão arterial sistêmica, aumento da agregação plaquetária, hipercoagulabilidade sangüínea, disfunção endotelial, dentre outros. Além disso, durante a apnéia ocorre um decréscimo no fluxo sangüíneo cerebral secundário à redução no débito cardíaco, que pode predispor indivíduos de risco ao AVC, como os portadores de lesões ateromatosas de circulação carotídea e vertebral. Isso pode ser significantemente mais importante durante o sono REM, estado em que há maior demanda de oxigênio encefálico.

Além disso, a apnéia pode comprometer a cognição, nos pacientes com história de AVC prévio, por determinar aumento da sonolência diurna, prejuízo na concentração e memória ${ }^{78}$. Por fim, estudos clínicos randomizados avaliando o tratamento da apnéia e evolução neurológica ainda não estão disponíveis.

\section{Insuficiência cardíaca congestiva}

Vários estudos também mostraram importante associação entre a insuficiência cardíaca congestiva (ICC) e SAHOS. Javaheri e cols. ${ }^{79}$ realizaram polissonografia em 81 homens portadores de ICC, identificando a síndrome em $11 \%$ dos pacientes. No estudo Sleep Heart Health ${ }^{80}$, detectou-se que em pacientes apnéicos com IAH > 11/hora o risco para desenvolver ICC foi de 2,38, independentemente de outros fatores de risco estabelecidos, excedendo os encontrados para outras complicações cardiovasculares da SAHOS, como hipertensão, DAC e AVC.

Acredita-se também que a ICC pode colaborar no desenvolvimento da SAHOS por dois motivos: diminuição do tônus muscular das vias aéreas superiores durante a fase de repouso da respiração periódica típica da ICC, e acúmulo de fluidos em partes moles da região cervical, facilitando a tendência ao colapso das vias aéreas superiores.

\section{REFERÊNCIAS}

1. Murray CJL, Lopez AD. The global burden of disease: a comprehensive assessment of mortality and disability from disease, injuries and risk factors in 1990 and projected to 2020. USA. Harvard School of Health; 1996.
Estudos com ecocardiografia demonstraram disfunção tanto sistólica ${ }^{81}$ quanto diastólica ${ }^{82}$ com aumento do $\mathrm{IAH}$. Possíveis mecanismos incluem efeitos da hipóxia (determinando isquemia e prejuízo da contratilidade), injúria dos miócitos (pelo aumento da concentração das catecolaminas circulantes) e mudança repetitiva nas pressões intratorácicas que acompanham os episódios de apnéia (alterando o relaxamento e o volume sistólico e diastólico final do ventrículo esquerdo)

Finalmente, o diagnóstico dos distúrbios respiratórios do sono em pacientes portadores de ICC pode trazer importantes informações prognosticas e uma opção terapêutica potencial para esse grupo de pacientes ${ }^{83}$.

\section{Alterações inflamatórias, endoteliais e trombóticas na SAHOS}

Foi demonstrada diminuição ou ausência de mecanismos fisiológicos de relaxamento vascular em resposta a substâncias endotélio-dependentes (aumento do vasoconstritor, endotelina e redução da disponibilidade do ácido nítrico e prostaciclinas) ${ }^{84,85}$. Outra evidência de lesão endotelial foi documentada mediante a produção aumentada de moléculas de adesão, levando a maior ligação de monócitos ao endotélio ${ }^{86}$. A SAHOS pode também desencadear 0 estresse oxidativo recorrente ${ }^{87}$, provavelmente em razão da produção aumentada de espécies oxigênio-reativas por monócitos e granulócitos. Esses achados reforçam resultados prévios de uma produção aumentada de radicais superóxidos por polimorfonucleares em pacientes com $\mathrm{SAHOS}^{88}$ e baixos níveis de óxido nítrico ${ }^{89}$. A homocisteína também foi descrita como aumentada nessa síndrome ${ }^{90}$, como resultado de disfunção endotelial combinada com excessiva formação de radicais livres, existindo também relatos de aumento de haptoglobina e proteína amilóide sérica $A^{91,92}$.

A ligação entre o processo inflamatório e as doenças cardiovasculares tem sido postulada repetidamente ${ }^{93}$. Pacientes com SAHOS são expostos cronicamente a hipóxia recorrente e a fragmentação do sono, apresentando níveis aumentados de proteína C-reativa e interleucina 6 , quando comparados com controles ${ }^{94}$, existindo também a possibilidade do aumento de proteína C-reativa em tais pacientes por causa da obesidade associada ${ }^{95}$. Todas essas alterações endoteliais, da coagulação sangüínea e inflamatórias podem contribuir de algum modo para o aumento do risco cardiovascular e aterogênese em pacientes com SAHOS. Por fim, observase que o tratamento com CPAP é capaz de melhorar essas alterações, ocorrendo retorno das anormalidades com a suspensão da terapêutica ${ }^{96-103}$.

2. Lolio CA, Laurenti R. Evolução da mortalidade por doença isquêmica do coração no município de São Pauo, 1970 a 1981. Arq Bras Cardiol. 1986; $46: 153$ 
3. Uemura K, Pisa Z. Recent trends in cardiovascular disease mortality in 27 industrialized countries. WId HIth Stat Quart. 1971; 38: 161725 .

4. Kannel WB, D'Agostino RB, Wilson PW, Belanger AJ, Gagnon DR. Diabetes, fibrinogen, and risk of cardiovascular disease: the Framingham experience. Am Heart J. 1990; 120(3): 672-6.

5. Kannel WB. Lessons from curbing the coronary artery disease epidemic for confronting the impending epidemic of heart failure. Med Clin North Am. 2004; 88: 1129-33.

6. Avezum A, Piegas LS, Pereira JC. Fatores de risco associados com infarto agudo do miocárdio na região metropolitana de São Paulo. Uma região desenvolvida em um país em desenvolvimento. Arq Bras Cardiol. 2005; 84: 206-13.

7. Marin JM, Carrizo SJ, Vicente E, Agusti AGN. Long-term cardiovascular outcomes in men with obstructive sleep apnea-hypopnoea with or without treatment with continuous positive airway pressure: an observational study. Lancet. 2005; 365: 1046-53.

8. Kramer NR, Cook TE, Carlisle CC, Corwin RW, Millman RP. The role of the primary care physician in recognizing obstructive sleep apnea. Arch Intern Med. 1999; 159: 965-8.

9. Young T, Palta M, Dempsey J, Skatrud J, Weber S, Badr S. The occurrence of sleep-disordered breathing among middle-age adults. N Engl Med. 1993; 328: 1230-35.

10. Olson LG, King MT, Hensley MJ, Saunders NA. A community study of snoring and sleep-disordered breathing: Prevalence. Am J Respir Crit Care Med. 1995; 152: 711-6.

11. Lavie P. Sleep apnea in industrial workers. In Guilleminault C, Lugaresi E, editors. Sleep-wake disorders: natural history, epidemiology and long-term evolution. Philadelphia: Lippincott Raven Press; 1983: 127-35.

12. Stradling JR, Crosby JH. Predictors and prevalence of obstructive sleep apnoea and snoring in 1001 middle aged men. Thorax. 1991; 46: 85-90.

13. Gislason T, Benediktsdottir B, Bjornsson JK, Kjartansson G, Kjeld M, Kristbjarnarson H. Snoring, hypertension, and sleep apnea syndrome: an epidemiologic survey of middle-aged women. Chest. 1993; 103: 1147-51

14. Bixler EO, Kales A, Soldatos CR, Vela-Bueno A, Jacoby JA, Scarone S. Sleep apneic activity in a normal population. Res Commun Chem Pathol Pharmacol. 1982; 36: 141-52.

15. Strohl KP, Redline S. Recognition of obstructive sleep apnea. Am J Respir Crit Care Med. 1996; 154: 279-89.

16. Silverberg DS, Oksenberg A. Are sleep-related breathing disorders important contributing factors to the production of essential hypertension? Curr Hypertens Rep. 2001; 3: 209-15.

17. EEG arousals: scoring rules and examples. A preliminary report from Sleep Disorders Atlas Task Force of the American Sleep Disorders Association. Sleep. 1992; 15: 173-84.

18. Rechtschaffen A, Kales A. A manual of standardized terminology, technique and scoring system for sleep stages of human sleep, Los Angeles Brain Information Service, Brain Information Institute, Los Angeles, CA; 1968.

19. Sleep-Related Breathing Disorders in Adults: Recommendations for syndrome definition and measurement techniques in clinical research. The report of an American Academy of Sleep Medicine Task Force. Sleep. 1999; 22: 667-89

20. Remmers JE, deGroot WJ, Sauerland EK, Anch AM. Pathogenesis of upper airway occlusion during sleep. J Appl Physiol. 1978; 44(6): 931-8.

21. Wiegand L, Zwillich CW. Obstructive sleep apnea. Dis Mon. 1994; 40(4): 197-252.
22. Strohl KP, Novak RD, Singer W, Cahan C, Boehm KD, Denko CW, Hoffstem VS. Insulin levels, blood pressure and sleep apnea. Sleep. 1994; 17(7): 614-8.

23. McCord JM. Oxygen-derived free radicals in postischemic tissue injury. N Engl J Med. 1985; 312(3): 159-63.

24. Findley LJ, Boykin M, Fallon T, Belardinelli L. Plasma adenosine and hypoxemia in patients with sleep apnea. J Appl Physiol. 1988; 64(2): 556-61.

25. Guilleminault C. State of the art. Sleep and control of breathing. Chest. 1978; 73(2): 293, 297-9.

26. Vgontzas AN, Bixler EO, Chrousos GP. Sleep apnea is a manifestation of the metabolic syndrome. Sleep Med Rev. 2005;9(3):211-24.

27. Yun AJ, Lee PY, Bazar KA. Autonomic dysregulation as a basis of cardiovascular, endocrine, and inflammatory disturbances associated with obstructive sleep apnea and other conditions of chronic hypoxia, hypercapnia, and acidosis. Med Hypotheses. 2004; 62(6): 852-6.

28. Opp MR, Kapas L, Toth LA. Cytokine involvement in the regulation of sleep. Proc Soc Exp Biol Med. 1992; 201(1): 16-27.

29. Vgontzas AN, Papanicolaou DA, Bixler EO, Kales A, Tyson K, Chrousos GP. Elevation of plasma cytokines in disorders of excessive daytime sleepiness: role of sleep disturbance and obesity. J Clin Endocrinol Metab. 1997; 82(5): 1313-6.

30. Fried SK, Bunkin DA, Greenberg AS. Omental and subcutaneous adipose tissues of obese subjects release interleukin-6: depot difference and regulation by glucocorticoid. J Clin Endocrinol Metab. 1998; 83(3): 847-50.

31. Vgontzas AN, Bixler EO, Chrousos GP. Metabolic disturbances in obesity versus sleep apnoea: the importance of visceral obesity and insulin resistance. J Intern Med. 2003; 254(1): 32-44.

32. Coy TV, Dimsdale JE, Ancoli-Israel S, Clausen J. Sleep apnoea and sympathetic nervous system activity. J Sleep Res. 1996; 5: 42-50.

33. Guilleminault C, Poyares D, Rosa A, Huang YS. Heart rate variability, sympathetic and vagal balance and EEG arousals in upper airway resistance and mild obstructive sleep apnea syndromes. Sleep Med. 2005; 6(5): 451-7.

34. Poyares D, Guilleminault C, Rosa A, Ohayon M, Koester U. Arousal, EEG spectral power and pulse transit time in UARS and mild OSAS subjects. Clin Neurophysiol. 2002; 113(10): 1598-606.

35. Smith ML, Neidermaier ON, Hardy SM, Decker MJ, Strohl KP. Role of hypoxemia in sleep apnea-induced sympathoexcitation. J Auton Nerv Syst. 1996; 56: 184-90.

36. Morgan BJ, Carbtree DC, Palta M, Skatrud J. Combine hypoxia and hypercapnia evokes long-lasting sympathetic activation in humans. J Appl Physiol. 1995; 79(1): 205-13.

37. Parati G, Di Rienzo M, Bonsignore MR, Insalaco G, Marrone O, Castiglioni $\mathrm{P}$, et al. Autonomic cardiac regulation in obstructive sleep apnea syndrome: evidence from spontaneous baroreflex analyss during sleep. J Hypertension. 1997; 15: 1621-6.

38. Jo JA, Blasi A, Valladares E, Juarez R, Baydur A, Khoo MC. Model-based assessment of autonomic control in obstructive sleep apnea syndrome during sleep. Am J Respir Crit Care Med. 2003; 167(2):128-36.

39. Arabi Y, Morgan BJ, Goodman B, Puleo DS, Xie A, Skatrud JB. Daytime blood pressure elevation after nocturnal hypoxia. J Appl Physiol. 1999; 87(2): 689-98

40. Xie A, Skatrud JB, Puleo DS, Morgan BJ. Exposure to hypoxia produces long-lasting sympathetic activation in humans. J Appl Physiol. 2001; 91(4): 1555-62.

41. Belozeroff $\mathrm{V}$, Berry RB, Khoo MC. Model-based assessment of autonomic control in obstructive sleep apnea syndrome. Sleep. 2003; 26(1): 65-73. 
42. Nieto FJ, Young TB, Lind BK, Shahar E, Samet JM, Redline S, et al. Association of sleep-disordered breathing, sleep apnea, and hypertension in a large community-based study. Sleep Heart Health Study. JAMA. 2000; 283(14): 1829-36.

43. Peppard PE, Young T, Palta M, Skatrud J. Prospective study of the association between sleep-disordered breathing and hypertension. $\mathrm{N}$ Engl J Med. 2000; 342(19): 1378-84.

44. Hedner J. Regulation of systemic vasculature in obstructive sleep apnea syndrome. Sleep. 2000; 23(Suppl 4): S132-5.

45. Lavie $P$, Herer P, Hoffstein V. Obstructive sleep apnoea syndrome as a risk factor for hypertension: population study. BMJ. 2000; 320(7233): 479-82.

46. Sin DD, Fitzgerald F, Parker JD, Newton GE, Logan AG, Floras JS, et al. Relationship of systolic BP to obstructive sleep apnea in patients with heart failure. Chest. 2003; 123(5): 1536-43.

47. Silverberg DS, Oksenberg A, laina A. Sleep related breathing disorders are common contributing factors to the production of essential hypertension but are neglected, underdiagnosed, and undertreated. Am J Hypertens. 1997; 10(12 Pt 1): 1319-25.

48. Shahar E, Whitney CW, Redline S, Lee ET, Newman AB, Javier Nieto F, O'Connor GT, Boland LL, Schwartz JE, Samet JM. Sleep-disordered breathing and cardiovascular disease - Cross-sectional results of the Sleep Heart Study. Am J Respir Crit Care Med. 2001; 163: 19-25.

49. Logan AG, Perlikowski SM, Mente A, Tisler A, Tkacova R, Niroumand $M$, et al. High prevalence of unrecognized sleep apnoea in drugresistant hypertension. J Hypertension. 2001; 19(12): 2271-7.

50. Fletcher EC. The relationship between systemic hypertension and obstructive sleep apnea: facts and theory. Am J Med. 1995; 98(2): $118-28$.

51. Hla KM, Skatrtud JB, Finn L, Palta M, Young T. The effect of correction of sleep-disordered breathing on BP in untreated hypertension. Chest. 2002; 122(4): 1111-2

52. Sanner BM, Tepel M, Markmann A, Zidek W. Effect of continuous positive airway pressure theraphy on 24-hour blood pressure in patientes with obstructive sleep apnea syndrome. Am J Hypertens. 2002; 15(3): 251-7.

53. Logan AG, Tkacova R, Perlikowski SM, Leung RS, Tisler A, Floras JS, et al. Refractory hypertension and sleep apnoea: effect of CPAP on blood pressure and baroreflex. Eur Respir J. 2003; 21(2): 241-7.

54. Hedner J, Darpo B, Ejnell H, Carlson J, Caidahl K. Reduction in sympathetic activity after long-term CPAP treatment in sleep apnoea cardiovascular implications. Eur Respir J 1995; 8: 222-9.

55. Somers VK, Dyken ME, Clary MP, Abboud FM. Sympathetic neural mechanisms in obstructive sleep apnea. J Clin Invest. 1995; 96(4): 1897-904.

56. Pepperell JC, Davies RJ, Stradling JR. Systemic hypertension and obstructive sleep apnoea. Sleep Med Rev. 2002; 6(3): 157-73.

57. Miller WP. Cardiac arrhythmias and conduction disturbances in the sleep apnea syndrome. Prevalence and significance. Am J Med. 1982; 73: 317-21.

58. Guilleminault C, Connoly SJ, Winkle RA. Cardiac arrhythmias and conduction disturbances during sleep in 400 patients with sleep apnea syndrome. Am J Cardiol. 1983; 52: 490-4.

59. Javaheri S. Effects of continuous positive airway pressure on sleep apnea and ventricular irritability in patients with heart failure. Circulation. 2000; 101: 392-7.

60. Garrigue S, Bordier P, Jais P, Shah DC, Hocini M, Raherison C, et al. Benefit of atrial pacing in sleep apnea syndrome. N Engl J Med. 2002; 346: 404-12

61. Mooe T, Gullsby S, Rabben T, Eriksson P. Sleep-disordered breathing: a novel predictor of atrial fibrillation after coronary artery bypass surgery. Coron Artery Dis. 1996; 7: 475-8.

62. Kanagala R, Murali NS, Friedman PA, Ammash NM, Gersh BJ, Ballman $\mathrm{KV}$, et al. Obstructive Sleep Apnea and recurrence of atrial fibrillation. Circulation. 2003; 107: 2589-94

63. Schulz R, Eisele HJ, Seeger W. Nocturnal atrial fibrillation in a patient with obstructive sleep apnoea. Thorax. 2005; 60(2): 174

64. Gami AS, Pressman G, Caples SM, Kanagala R, Gard JJ, Davison DE, et al. Association of atrial fibrillation and obstructive sleep apnea. Circulation. 2004; 110(4): 364-7

65. Porthan KM, Melin JH, Kupila JT, Venho KK, Partinen MM. Prevalence of sleep apnea syndrome in lone atrial fibrillation: a case-control study. Chest. 2004; 125(3): 879-85.

66. Flemons WW, Remmers JE, Gillis AM. Sleep apnea and cardiac arrhythmias. Is there a relationship? Am Rev Respir Dis. 1993; 148: 618-21.

67. Roche F, Xuong ANT, Court-Fortune I, Costes F, Pichot V, Duverney D, et al. Relationship among severity of sleep apnea syndrome, cardiac arrhythmias, and autonomic imbalance. PACE. 2003; 26: 669-77.

68. Shepard JW Jr, Garrison MW, Grither DA, Dolan GF. Relationship of ventricular ectopy to oxyhemoglobin desaturation in patients with obstructive sleep apnea. Chest. 1985; 88: 335-440.

69. Cutler MJ, Hamdan AL, Hamdan MH, Ramaswamy K, Smith ML. Sleep apnea: from nose to the heart. J Am Board Farm Pract. 2002; 15: 128-41.

70. Guilleminault C, Connolly SJ, Winkle RA. Cardiac arrhythmia and conduction disturbances during sleep in 400 patients with sleep apnea syndrome. Am J Cardiol. 1983; 52: 490-4.

71. Koehler U, Fus E, Grimm W, Pankow W, Schafer H, Stammnitz A, et al. Heart block in patients with obstructive sleep apnoea: pathogenetic factors and effects of treatment. Eur Respr J. 1998; 11: 434-9.

72. Carlson JT, Hedner J, Elam M, Ejnell H, Sellgren J, Wallin BG. Augmented resting sympathetic activity in awake patients with obstructive sleep apnea. Chest. 1993; 103: 1763-8.

73. Libby P, Ridker PM, Maresi A. Inflammation and atherosclerosis. Circulation. 2002; 105: 1135-43.

74. Milleron O, Pilliere R, Foucher A, de Roquefeuil F, Aegerter P, Jondeau $G$, et al. Benefits of obstructive sleep apnoea treatment in coronary artery disease: a long-term follow-up study. Eur Heart J. 2004; 25(9): 728-34.

75. Mooe T, Franklin KA, Wiklund U, Rabben T, Holmstrom K. Sleepdisordered breathing and myocardial ischemia in patients with coronary artery disease. Chest. 2000; 117(6): 1597-602.

76. Bassetti CL. Sleep and stroke. Semin Neurol. 2005; 52(1): 19-32.

77. Dziewas R, Humpert M, Hopmann B, Kloska S, Ludemann P, Ritter M, et al. Increased prevalence of sleep apnea in patients with recurring ischemic stroke compared with first stroke victims. J Neurol. 2005; 252 (11): 1394-8; Epub 2005 Jul 20.

78. Engleman H, Joffe D. Neuropsychological function in obstructive sleep apnoea. Sleep Med Rev. 1999; 3(1): 59-78.

79. Javaheri S, Parker TJ, Liming JD, et al. Sleep apnea in 81 ambulatory male patients with stable heart failure: types and their prevalences, consequences, and presentations. Circulation. 1998; 97: 2154-9.

80. Shahar E, Whitney CW, Redline S, Lee ET, Newman AB, Javier Nieto $F$, et al. Sleep-disordered breathing and cardiovascular disease: crosssectional results of the Sleep Heart Health Study. Am J Respir Crit Care Med. $2001 ; 163(1)$ : 19-25.

81. Laaban JP, Pascal-Sebaoun S, Bloch E, Orvoen-Frija E, Oppert JM, Huchon G. Left ventricular systolic dysfunction in patients with obstructive sleep apnea syndrome. Chest. 2002; 122: 1133-8. 
82. Fung JW, Li TS, Choy DK, Yip GW, Ko FW, Sanderson JE, et al. Severe obstructive sleep apnea is associated with left ventricular diastolic dysfunction. Chest. 2002; 121: 422-9

83. Wilcox I, McNamara SG, Wessendorf T, Willson GN, Piper AJ, Sullivan CE. Prognosis and sleep disordered breathing in heart failure. Thorax. 1998; 53(3): S33-6.

84. Fletcher EC. Sympathetic over activity in the etiology of hypertension of obstruvtive sleep apnea. Sleep. 2003; 26(1): 15-9.

85. Pepin JI, Levy P. Pathophysiology of cardiovascular risk in sleep apnea syndrom (SAS). Rev Neurol. 2002; 158: 785-97.

86. Dyugovskaya L, Lavie P, Lavie L. Increased adhesion molecules expression and production of reactive oxygen species in leukocytes of sleep apnea patients. Am J Respir Crit Care Med. 2002; 165(7): 934-9.

87. Lavie L. Obstructive sleep apnoea syndrome - an oxidative stress disorder. Sleep Med Rev. 2003; 7(1): 35-51.

88. Schulz R, Mahmoudi S, Hattar K, Sibelius U, Olschewski H, Mayer $\mathrm{K}$, et al. Enhanced release of superoxide from polymorphonuclear neutrophils in obstructive sleep apnea. Impact of continuous airway pressure therapy. Am J Respir Crit Care Med. 2000; 162: 566-8.

89. Ip MS, Lam B, Chan LY, Zheng L, Tsang KW, Fung PC, et al. Circulation nitric oxide is suppressed in obstructive sleep apnea and is reversed by nasal continuous positive airway pressure. Am J Respir Crit Care Med. 2000; 162: 2166-71.

90. Lavie L, Perelman A, Lavie P. Plasma homicysteine levels in obstructive sleep apnea patients. Chest. 2001; 120: 900-8.

91. Lavie L, Lotan R, Hochberg I, Herer P, Lavie P, Levy AP. Haptoglobin polymorphism is a risk factor for cardiovascular disease in patients with obstructive sleep apnea syndrome. Sleep. 2003; 26(5): 592-5.

92. Svatikova A, Wolk R, Shamsuzzaman AS, Kara T, Olson EJ, Somers VK. Serum amyloid a in obstructive sleep apnea. Circulation. 2003; 108(12): 1451-4.

93. Ross R. Atherosclerosis - an inflamatory disease. N Engl J Med. 1999; 340: 115-26.
94. Yokoe T, Minoguchi K, Matsuo H, Oda N, Minoguchi H, Yoshino G, et al. Elevated levels of $\mathrm{C}$-reactive protein and interleukin- 6 in patients with obstructive sleep apnea syndrome are decreased by nasal continuous positive airway pressure. Circulation. 2003; 107(8):1129-34.

95. Guilleminault C, Kirisoglu C, Ohayon MM. C-reactive protein and sleep-disordered breathing. Sleep. 2004; 15; 27(8): 1507-11.

96. Kato M, Roberts-Thomson P, Phillips BG, Haynes WG, Winnicki M, Accurso $\mathrm{V}$, et al. Impairment of endothelium-dependent vasodilation of resistance vessels in patients with obstructive sleep apnea. Circulation. 2000; 21; 102(21): 2607-10.

97. Kraiczi H, Caidahl K, Samuelsson A, Peker Y, Hedner J. Impairment of vascular endothelial function and left ventricular filling: association with the severity of apnea-induced hypoxemia during sleep. Chest. $2001 ; 119(4): 1085-91$

98. Teramoto S, Kume $\mathrm{H}$, Yamamoto $\mathrm{H}$, Ishii T, Miyashita A, Matsuse $T$, et al. Effects of oxygen administration on the circulating vascular endothelial growth factor (VEGF) levels in patients with obstructive sleep apnea syndrome. Intern Med. 2003; 42(8): 681-5.

99. Shamsuzzaman AS, Gersh BJ, Somers VK. Obstructive sleep apnea: implications for cardiac and vascular disease.JAMA. 2003; 290(14): 1906-14.

100. Nieto FJ, Herrington DM, Redline S, Benjamin EJ, Robbins JA. Sleep apnea and markers of vascular endothelial function in a large community sample of older adults. Am J Respir Crit Care Med. 2004; 169(3): 354-60.

101. Dyugovskaya L, Lavie P, Lavie L. Phenotypic and functional characterization of blood gammadelta T cells in sleep apnea. Am J Respir Crit Care Med. 2003; 168(2): 242-9.

102. Schulz R, Hummel C, Heinemann S, Seeger W, Grimminger F. Serum levels of vascular endothelial growth factor are elevated in patients with obstructive sleep apnea and severe nighttime hypoxia. Am J Respir Crit Care Med. 2002; 165(1): 67-70

103. Von Kanel R, Le DT, Nelesen RA, Mills PJ, Ancoli-Israel S, Dimsdale $\mathrm{JE}$. The hypercoagulable state in sleep apnea is related to comorbid hypertension. J Hypertens. 2001; 19(8): 1445-51. 